\title{
Analyst
}

PAPER

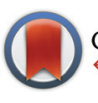

CrossMark \&lick for updates

Cite this: Analyst, 2015, 140, 1453

\section{Controlled growth of immunogold for amplified optical detection of aflatoxin B1}

\author{
Xu Wang, Reinhard Niessner and Dietmar Knopp*
}

A simple, sensitive and cost-effective method for the analysis of the mycotoxin aflatoxin B1 (AFB1) has been established based on controlled growth of immunogold. AFB1-BSA conjugate modified magnetic beads were employed as capture probe and anti-AFB1 antibody-coated gold colloids were used as detection probe for the immunological recognition of AFB1, as well as for signal transduction. The immune recognition event is converted into the gold enlargement signal which can be quantitatively measured by UV-vis spectroscopy. The autocatalytic enlargement of immunogold was conducted in aqueous solution containing chloroauric acid, hexadecyltrimethylammonium bromide and ascorbic acid. The reaction could be stopped by the addition of sodium thiosulfate. The final absorbance and resonance light scattering intensity were highly dependent on immunogold concentration. After gold enhancement, the sensitivity of the immunoassay was improved and total assay time reduced to $1 \mathrm{~h}$. Under optimized conditions, the linear range and lower detection limit was $0.01-1 \mathrm{ng} \mathrm{mL}^{-1}$ and $7 \mathrm{pg} \mathrm{mL}^{-1}$, respectively. The proposed method offers great promise for sensitive detection of other mycotoxins and organic pollutants.

Received 12th December 2014 Accepted 12th January 2015

DOI: $10.1039 / c 4 a n 02281 \mathrm{e}$ www.rsc.org/analyst resulting in obvious absorbance change, which can be easily read out with the naked eye or special analytical equipment. As another example, AuNPs served as seeds for silver enlargement where the reduction and deposition of silver ions are catalyzed by the AuNPs. ${ }^{18}$

The so-called silver staining method has been well developed and extensively applied in biomolecular detection. ${ }^{19}$ However, as a disadvantage, the silver salt is usually sensitive to $\mathrm{pH}$, phosphates, chloride ions and natural light, which limits its practical application. As an alternative, the gold staining, i.e., using gold salt for catalytic enlargement of AuNPs, has solved these problems. The gold staining methods have been widely used for the detection of various targets such as proteins and DNA. ${ }^{20-22}$ However, this technique is mostly applied onto solid substrates like glass slides ${ }^{23}$ and nitrocellulose strips ${ }^{20}$ therefore, strict control of the reaction conditions are required to obtain acceptable reproducibility. Several homogeneous detection formats have been developed to solve this problem. ${ }^{24-26}$ The AuNPs were enlarged in aqueous solution, which shows some attractive attributes such as simpleness, low cost and high sensitivity. But generally, a long reaction time is required for homogeneous gold nanogrowth. ${ }^{26}$ Since the gold enlargement is time-dependent, the reaction continues until all the gold ions in growth solution are exhausted. Significantly, we recently found that sodium thiosulfate can efficiently stop the reaction, which makes the homogenous gold staining more controllable and convenient.

In this work, controlled growth of AuNPs in aqueous solution was studied in-depth by UV-vis spectroscopy and
Institute of Hydrochemistry, Chair for Analytical Chemistry, Technische Universität München, Marchioninistrasse 17, D-81377 München, Germany.

E-mail: dietmar.knopp@ch.tum.de; Fax: +49-89-2180-78255;

Tel: $+49-89-2180-78252$ 


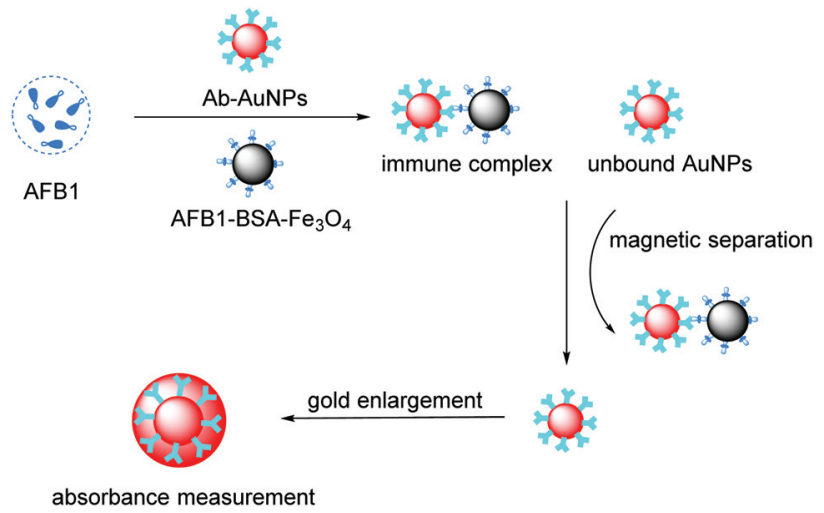

Fig. 1 Schematic illustration of the homogeneous gold staining for amplified optical detection of AFB1.

resonance light scattering (RLS) technique, using hexadecyltrimethylammonium bromide (CTAB) as stabilizing surfactant and ascorbic acid (AA) as reducing agent. The controlled gold staining was then used for signal amplification in competitive immunoassay (Fig. 1). The mycotoxin aflatoxin B1 (AFB1) was chosen as the model analyte. ${ }^{27}$ Although various methods have been developed for AFB1 detection, such as instrumental analysis using high-performance liquid chromatography (HPLC) ${ }^{28}$ or liquid chromatography coupled to mass spectrometry $(\mathrm{LC}-\mathrm{MS})^{29}$ and different immunological assays, ${ }^{30-32}$ even more simple and rapid methods are still desirable. AFB1-BSA conjugate modified magnetic beads (AFB1-BSA-Fe ${ }_{3} \mathrm{O}_{4}, \mathrm{MBs}$ ) were employed as capture probe, while anti-AFB1 antibody-coated AuNPs (Ab-AuNPs) were used as detection probe for immunological recognition of AFB1, as well as for signal transduction. Since MBs were removed from the reaction mixture, they did not participate in the subsequent gold enlargement. After signal amplification, the sensitivity of the immunoassay increased distinctly. To the best of our knowledge, this is the first time that homogeneous gold staining was applied to a competitive immunoassay.

\section{Experimental}

\section{Materials}

Chloroauric acid $\left(\mathrm{HAuCl}_{4}\right)$, hexadecyltrimethylammonium bromide (CTAB), ascorbic acid (AA), sodium thiosulfate $\left(\mathrm{Na}_{2} \mathrm{~S}_{2} \mathrm{O}_{3}\right.$ ), Tween-20, ochratoxin A (OTA), T-2 toxin, fumonisin B1 (FB1) and AFB1 were purchased from Sigma Aldrich (Taufkirchen, Germany). Disodium hydrogen phosphate $\left(\mathrm{Na}_{2} \mathrm{HPO}_{4}\right)$ and sodium dihydrogen phosphate $\left(\mathrm{NaH}_{2} \mathrm{PO}_{4}\right)$ were purchased from Fluka (Buchs, Switzerland). Polyethylene glycol 8000 (PEG-8000) was obtained from Carl Roth (Karlsruhe, Germany). Phosphate buffer solution (PBS) was prepared by using $0.2 \mathrm{M} \mathrm{NaH}_{2} \mathrm{PO}_{4}$ and $0.2 \mathrm{M} \mathrm{Na}_{2} \mathrm{HPO}_{4}$ and then diluted to the corresponding concentration. The mouse monoclonal antiaflatoxin antibody $1 \mathrm{~F} 2$ was from our group. ${ }^{33}$ Ultrapure water was produced using reverse osmosis with UV treatment (MilliRO 5 Plus, Milli-Q185 Plus, Millipore, Eschborn, Germany).

\section{Apparatus}

UV-vis absorption spectra were measured on a Specord 250 Plus UV-vis spectrophotometer (Analytik Jena, Jena, Germany). RLS spectra were measured on a RF-5301 PC spectrofluorometer (Shimadzu, Tokyo, Japan) by simultaneously scanning the excitation and emission monochromators from 300 to $800 \mathrm{~nm}$ with $\Delta \lambda=0 \mathrm{~nm}$ and sensitivity set to low.

\section{Preparation of $\mathrm{Ab}-\mathrm{AuNPs}$ and $\mathrm{AFB1}-\mathrm{BSA}-\mathrm{Fe}_{3} \mathrm{O}_{4}$ magnetic beads}

Ab-AuNPs and MBs were prepared and characterized according to the published method. ${ }^{34}$ The AuNPs with size of $35 \mathrm{~nm}$ were used. The Ab-AuNPs were dispersed in $5 \mathrm{mM}$ PBS $(\mathrm{pH}$ 7.4) containing $0.1 \%$ PEG-8000. The concentration of AuNPs and MBs was estimated to be $0.40 \mathrm{nM}$ and $4 \mathrm{mg} \mathrm{mL}^{-1}$, respectively.

\section{Preparation of gold growth solution}

General procedure: $25 \mu \mathrm{L}$ of $0.1 \mathrm{M} \mathrm{HAuCl}_{4}$ was added to $10 \mathrm{~mL}$ of $100 \mathrm{mM}$ CTAB in water. The mixture was heated in a water bath and mixed until all the precipitates were dissolved. Then $100 \mu \mathrm{L}$ of $100 \mathrm{mM}$ AA solution was added. The solution changed immediately from clear orange to colorless. The growth solution was ready for use after cooling down to room temperature.

To study the influence of CTAB on gold enhancement, CTAB solution with different concentrations $(0,5,10,25,50$, $75,100 \mathrm{mM}$ ) was used to prepare the gold growth solution. To investigate the effect of AA, solutions were tested with different final concentrations $(0.5,0.55,0.6,0.75,1,2,5,10 \mathrm{mM})$. Volumes of 10,25 and $40 \mu \mathrm{L}$ of $\mathrm{HAuCl}_{4}$ were used to evaluate the influence of gold concentration.

\section{Growth of AuNPs in aqueous phase}

The immunogold nanoparticles $(\sim 0.20 \mathrm{nM}$ Ab-AuNPs in 25 mM PBS, pH 7.4, 0.05\% PEG-8000) were used as gold seeds for the enlargement. Generally, $50 \mu \mathrm{L}$ of gold seeds were added to $1.0 \mathrm{~mL}$ of the growth solution. The absorption spectra were measured after $30 \mathrm{~min}$. The kinetics of the AuNP growth was quantitatively monitored by UV-vis spectroscopy and RLS. To study the influence of immunogold concentration, different amounts of gold seeds $(5-50 \mu \mathrm{L})$ were added to $1.0 \mathrm{~mL}$ growth solution for gold enhancement.

\section{Analysis of maize samples}

Pulverized maize samples were purchased from supermarket in Munich. The maize samples ( $1 \mathrm{~g}$ ) were spiked with AFB1 at different concentrations $\left(0,4,8,20\right.$ and $\left.40 \mu \mathrm{g} \mathrm{kg} \mathrm{kg}^{-1}\right)$. The spiked samples were kept at room temperature under dark condition for $3 \mathrm{~h}$ to evaporate methanol used to prepare AFB1 standards and then extracted with $4 \mathrm{~mL}$ of methanol-water $(80: 20, \mathrm{v} / \mathrm{v}$ ) by vortex mixing for $2 \mathrm{~min}$ and then centrifugated at $1920 \mathrm{~g}$ for $15 \mathrm{~min}$. The supernatant was then 20 -fold diluted with PBS (50 mM, pH = 7.4) for quantitative analysis. 


\section{Assay procedure}

The AFB1 standard solutions with different concentrations were prepared in $50 \mathrm{mM}$ PBS ( $\mathrm{pH}$ 7.4). A volume of $50 \mu \mathrm{L}$ of AFB1 standards/samples was injected into a $0.5 \mathrm{~mL}$ Eppendorf tube, then $50 \mu \mathrm{L}$ of Ab-AuNPs and $15 \mu \mathrm{L}$ of MBs suspension $\left(\sim 4 \mathrm{mg} \mathrm{mL}^{-1}\right)$ were added successively. The mixture was well mixed and incubated under shaking for $30 \mathrm{~min}$. After removing the formed immune complexes (i.e. anti-AFB1-AuNP-AFB1BSA-MBs) by magnetic separation, $100 \mu \mathrm{L}$ of supernatant solution containing unbound AuNPs was transferred into $1.0 \mathrm{~mL}$ gold growth solution and the mixture was incubated at room temperature for $20 \mathrm{~min}$. Then $100 \mu \mathrm{L}$ of $10 \mathrm{mM} \mathrm{Na} \mathrm{S}_{2} \mathrm{O}_{3}$ was added to stop the reaction and the absorbance of the enlarged AuNPs was measured on UV-vis spectrophotometer. The absorbance at $565 \mathrm{~nm}$ (533 $\mathrm{nm}$ for AuNPs without gold staining) was recorded and final absorbance was calculated by subtracting the absorbance of the corresponding blank samples. Error bars were standard deviations across at least three repetitive assays.

\section{Results and discussion}

The principle of the immunoassay is illustrated in Fig. 1. Biofunctionalized MBs and free AFB1 molecules competitively bind to AuNP-labeled antibodies. After magnetic separation, the supernatant containing unbound AuNPs was directly submitted for gold enlargement. The final absorbance depends on the amount of immunogold nanoparticles added, which is directly proportional to the concentration of AFB1 in the sample.

\section{Proposed mechanism of gold enlargement}

The proposed mechanism of the homogeneous growth of AuNPs is illustrated in Fig. 2A. The immunogold nanoparticles
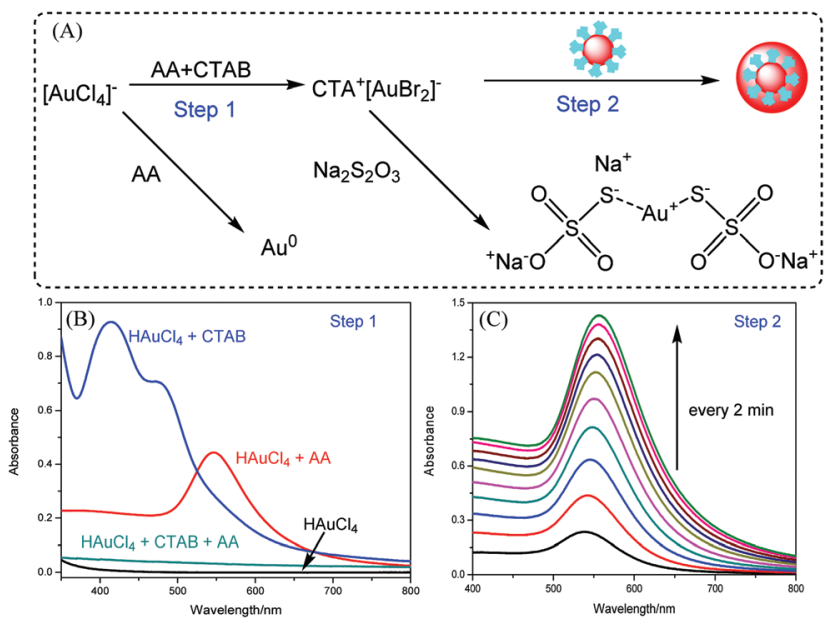

Fig. 2 (A) Proposed mechanism of gold enlargement in aqueous phase; (B) UV-vis absorption of $\mathrm{HAuCl}_{4}, \mathrm{HAuCl}_{4}+\mathrm{CTAB}, \mathrm{HAuCl}{ }_{4}+\mathrm{CTAB}+\mathrm{AA}$ and $\mathrm{HAuCl}_{4}+\mathrm{AA}$; (C) UV-vis absorption spectra of the enlarging AuNPs recorded every $2 \mathrm{~min}$. act as self-catalysts while the CTAB serves as a surfactant to stabilize the enlarged nanoparticles. Ascorbic acid was employed as the reducing agent for the gold enlargement.

In the first stage, the $\mathrm{Au}^{3+}$ ions were rapidly reduced to $\mathrm{Au}^{+}$ ions by $\mathrm{AA}$. The $\mathrm{Au}^{+}$ions can be stabilized by CTAB. As shown in Fig. 2B, chloroauric acid solution had very low absorption between $350 \mathrm{~nm}$ and $800 \mathrm{~nm}$. In the presence of CTAB, a significant absorption peak appeared at $416 \mathrm{~nm}$ with a shoulder around $470 \mathrm{~nm}$. This might be ascribed to ligand exchange between $\mathrm{HAuCl}_{4}$ and $\mathrm{CTAB}$. $\mathrm{Au}(\mathrm{III}) \mathrm{Br}_{4}$-CTA complex was formed, which takes on a clear orange color. ${ }^{35,36}$ After the addition of $\mathrm{AA}$, the $\mathrm{Au}(\mathrm{III}) \mathrm{Br}_{4}$-CTA complex was rapidly reduced to $\mathrm{Au}(\mathrm{I}) \mathrm{Br}_{2}$-CTA, which was colorless. In the absence of CTAB, the $\mathrm{Au}^{3+}$ ions were directly reduced to $\mathrm{Au}^{0}$. An obvious absorption peak was observed at $550 \mathrm{~nm}$, indicating the formation of small gold colloids. In the second stage, the Ab-AuNPs served as self-catalysts by receiving and transferring the electrons to $\mathrm{Au}^{+}$species. ${ }^{25}$ These $\mathrm{Au}^{+}$ions were then reduced to gold atoms which deposited onto the surfaces of Ab-AuNPs, resulting in the size growth of the AuNPs, which can be easily monitored by UV-vis spectroscopy, as indicated in Fig. 2C. The absorbance increased dramatically with increasing deposition time, while the absorption maximum was gradually red-shifted, which can be attributed to the increase of particle size and variations in the refractive index as well as partial aggregation of the AuNPs during enlargement.

\section{Controlled growth of immunogold in aqueous solution}

In order to optimize the gold enlargement, several experimental parameters were investigated in detail, including the concentrations of CTAB, AA and $\mathrm{HAuCl}_{4}$.

Typically, the CTAB used in this study not only stabilizes the reduced $\mathrm{Au}^{+}$ions but also is crucial for preventing the aggregation of enlarged AuNPs, which is very significant for assays based on optophysical properties of the nanostructures (e.g. absorption, light scattering). As shown in Fig. 3A, with the decrease of CTAB concentration, the absorption spectrum became broader and the maximum absorption was redshifted. This might be attributed to the enlarged AuNPs aggregated at low concentration of CTAB due to high surface energy. Thus, a high concentration of CTAB, $100 \mathrm{mM}$, was used in further experiments. The AA reduced $\mathrm{Au}^{3+}$ to $\mathrm{Au}^{+}$, and the $\mathrm{Au}^{+}$ species were further reduced to gold atoms in the presence of
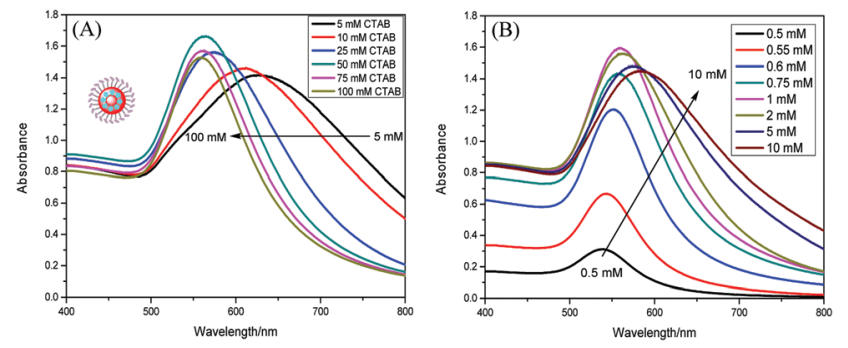

Fig. 3 Effect of (A) CTAB concentration $\left(0.25 \mathrm{mM} \mathrm{HAuCl}_{4}\right.$ and $1 \mathrm{mM}$ $\mathrm{AA})$ and (B) AA concentration (100 mM CTAB and $\left.0.25 \mathrm{mM} \mathrm{HAuCl}_{4}\right)$ on the absorbance of the enlarged AuNPs. 

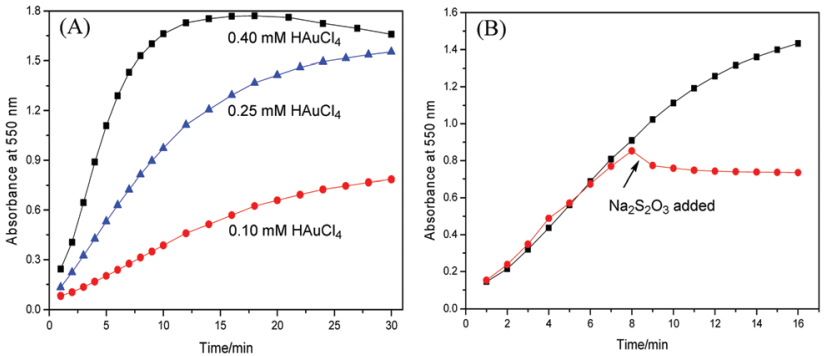

Fig. 4 Time-dependent absorbance changes at $550 \mathrm{~nm}$ of the enlarged AuNPs: (A) the effect of $\mathrm{HAuCl}_{4}$ concentration (100 mM CTAB and $1 \mathrm{mM}$ $\mathrm{AA})$ and (B) the effect of $\mathrm{Na}_{2} \mathrm{~S}_{2} \mathrm{O}_{3}(100 \mathrm{mM}$ CTAB, $1 \mathrm{mM} \mathrm{AA}$ and $0.25 \mathrm{mM}$ $\left.\mathrm{HAuCl}_{4}\right)$.

immunogold. A certain amount of AA was required to reduce all the gold ions. As shown in Fig. 3B, the absorbance increased rapidly when the AA concentration rose from 0.5 to $1 \mathrm{mM}$, and then decreased slightly in the range of $2-10 \mathrm{mM}$. The maximum absorbance occurred at $1 \mathrm{mM}$ AA. Therefore, $1 \mathrm{mM}$ AA was used for the following studies.

The influence of the concentration of $\mathrm{HAuCl}_{4}$ was also investigated as indicated in Fig. 4A. The absorbance at $550 \mathrm{~nm}$ was monitored by UV-vis spectroscopy. It is clearly seen that the gold enhancement was faster at higher concentration of $\mathrm{HAuCl}_{4}$. But at $0.4 \mathrm{mM} \mathrm{HAuCl}$, the absorbance decreased after 15 min. This might be caused by the enlarged AuNPs which are too big at high concentration of chloroauric acid and therefore, some particles precipitated, resulting in a decrease in the absorbance. Hence, $0.25 \mathrm{mM}$ of chloroauric acid was selected for the following experiments.

The gold enlargement is time-dependent and normally the process will be catalyzed continuously until all the gold ions are exhausted. Thus, a long reaction time is required. Recently, we found that $\mathrm{Na}_{2} \mathrm{~S}_{2} \mathrm{O}_{3}$ can efficiently stop the reaction. As shown in Fig. 4B, after the addition of $\mathrm{Na}_{2} \mathrm{~S}_{2} \mathrm{O}_{3}$, the absorbance did not increase anymore and kept almost constant. This is because stable gold-sodium thiosulfate complex was formed as shown in Fig. 2A, which could not be reduced by AA and is well soluble in water. Thus, $\mathrm{Na}_{2} \mathrm{~S}_{2} \mathrm{O}_{3}$ solution was used as stop solution for gold enlargement.

The homogeneous growth of immunogold was then conducted under optimized conditions. Fig. 5A shows the kinetics behavior of particle growth using different amounts of gold seeds. Various amounts of immunogold were added to $1.0 \mathrm{~mL}$ of growth solution and the absorbance was monitored. The absorption spectra after $30 \mathrm{~min}$ were shown in Fig. 5B. It can be clearly seen that the rate of gold enlargement and final absorption intensity are highly dependent on and proportional to the initial concentration of immunogold (Fig. 5C), which provides a quantitative basis for signal amplification.

\section{Monitoring the gold enlargement by RLS spectra}

The RLS spectra of enlarging AuNPs were also investigated. RLS occurs when the wavelength of incident light is close to that of the absorption band of metal nanoparticles. ${ }^{37}$ AuNPs

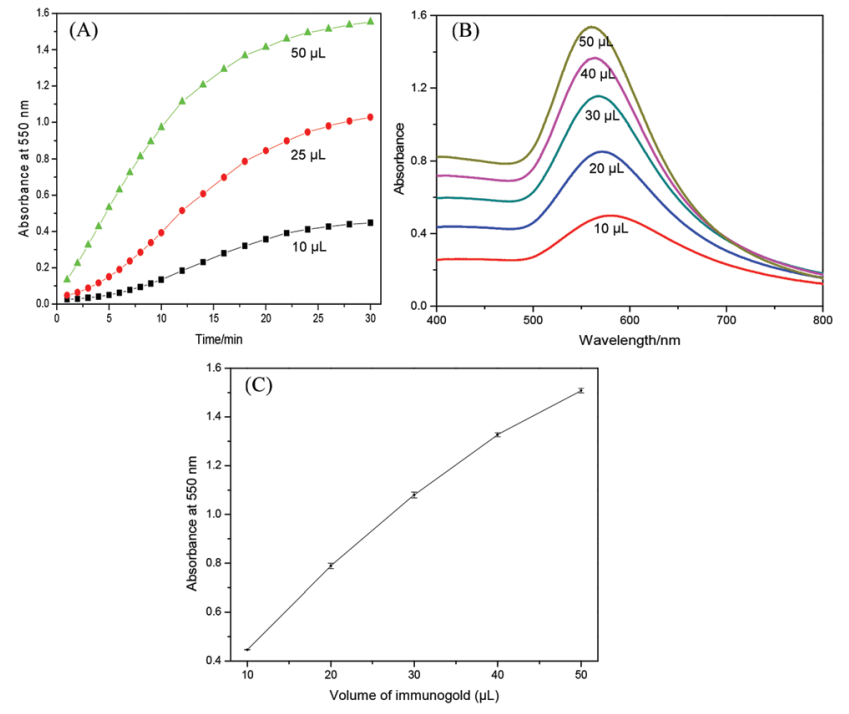

Fig. 5 (A) Time-dependent absorbance changes at $550 \mathrm{~nm}$ upon analyzing different amounts of immunogold nanoparticles; (B) UV-vis absorption spectra after deposition of $30 \mathrm{~min}$. (C) absorbance at $550 \mathrm{~nm}$ vs. the amount of immunogold. Conditions: different amounts of immunogold were added to $1 \mathrm{~mL}$ growth solution containing $100 \mathrm{mM} \mathrm{CTAB}$, $1 \mathrm{mM} \mathrm{AA}$ and $0.25 \mathrm{mM} \mathrm{HAuCl}_{4}$.
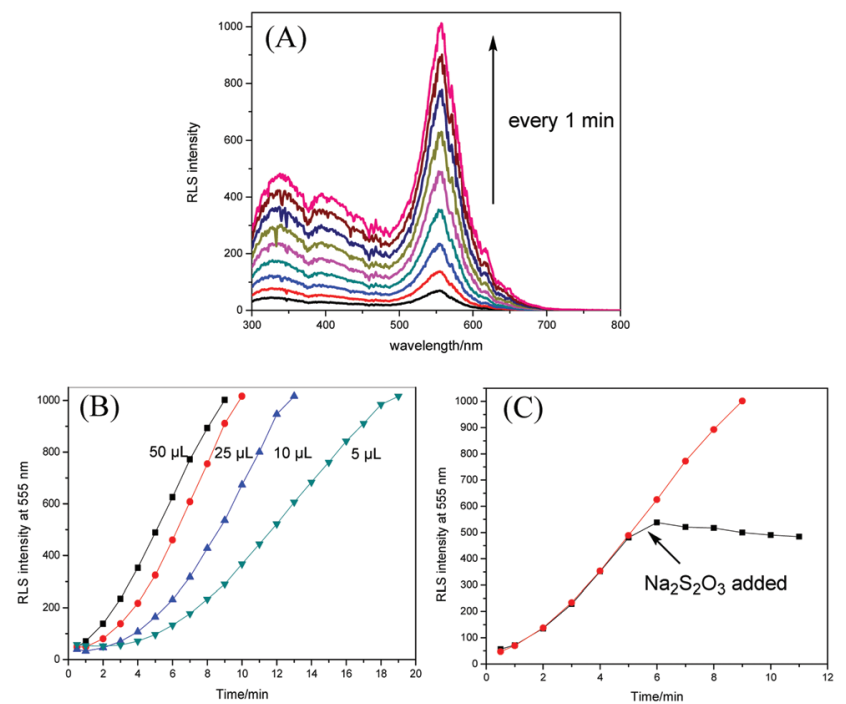

Fig. 6 (A) RLS spectra of the enlarging AuNPs recorded every 1 min; (B) Time-dependent RLS intensity changes at $555 \mathrm{~nm}$ upon analyzing different amounts of immunogold nanoparticles; (C) Effect of $\mathrm{Na}_{2} \mathrm{~S}_{2} \mathrm{O}_{3}$ on RLS intensity. Conditions: different amounts of immunogold were added to $1 \mathrm{~mL}$ growth solution containing $100 \mathrm{mM}$ CTAB, $1 \mathrm{mM}$ AA and $0.25 \mathrm{mM} \mathrm{HAuCl}_{4}$.

exhibit characteristic RLS peak at about $550 \mathrm{~nm}$. The RLS intensity can be greatly enhanced with increasing particle size. As shown in Fig. 6A, a typical RLS peak was observed around $555 \mathrm{~nm}$. With the increase of deposition time, the RLS intensity increased dramatically. Different from the absorption band, the wavelength of RLS peak kept almost the same. Fig. 6B shows the kinetic plots of RLS intensity at $555 \mathrm{~nm}$ 
versus time. The RLS intensity increased faster at higher concentration of immunogold. Similar to the absorption behavior, the RLS intensity did not increase when the gold enlargement was stopped by the addition of $\mathrm{Na}_{2} \mathrm{~S}_{2} \mathrm{O}_{3}$ (Fig. 6C). Thus, RLS technique could be utilized as another tool for monitoring the gold enhancement.

\section{Amplified optical detection of AFB1 through controlled growth of immunogold}

The AFB1 standards were quantitatively analyzed following the procedures described in the Experimental section. Fig. 7A shows the absorption spectra of supernatants after gold enhancement. With the increase of AFB1 concentration, the absorption intensity increased correspondingly, and the maximum absorption was slightly blue-shifted, which is ascribed to smaller enlarged AuNPs at higher concentration of immunogold. As seen in Fig. 7B, a linear dependence between absorbance change at $565 \mathrm{~nm}$ and AFB1 level could be achieved in the dynamic range from 0.01 to $1 \mathrm{ng} \mathrm{mL} \mathrm{m}^{-1}$ (ppb). The regression equation could be fitted to $y=0.4543 \times C_{\text {[AFB1] }}$ $+0.3182\left(\mathrm{ng} \mathrm{mL} \mathrm{m}^{-1}, R^{2}=0.988, n=6\right)$. The limit of detection (LOD) was estimated to be $0.007 \mathrm{ng} \mathrm{mL}^{-1}(7 \mathrm{ppt}$ ) based on three times of signal-to-noise ratio.

The detectable concentration range of the developed assay is comparable with that obtained by an ELISA ${ }^{38}$ or an electrochemical immunosensor, ${ }^{39}$ which used the same anti-AFB1 antibody. In addition, the sensitivity of proposed assay format is also high. Fig. 8 shows the comparison of AFB1 detection
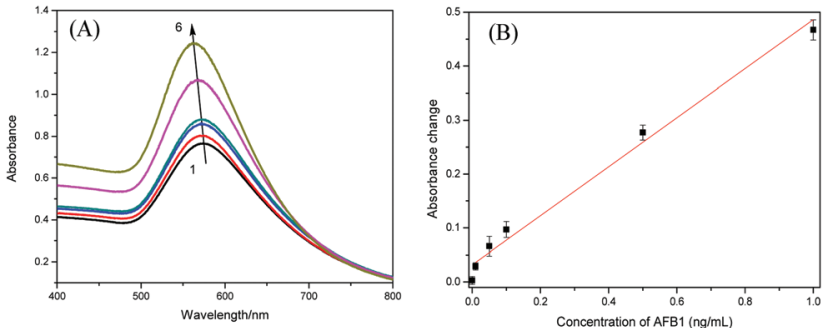

Fig. 7 (A) UV-vis absorption spectra of supernatant after gold enlargement with different concentrations of AFB1 (from sample 1 to 6: 0, 0.01, $0.05,0.1,0.5,1 \mathrm{ng} \mathrm{mL} \mathrm{m}^{-1}$ ); (B) The relationship between absorbance changes at $565 \mathrm{~nm}$ and AFB1 concentration $(n=6)$.

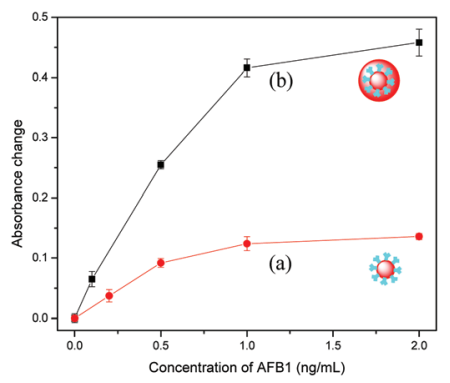

Fig. 8 Response curves of the developed immunoassay toward AFB1 standards: (a) without and (b) with gold enlargement $(n=3)$.

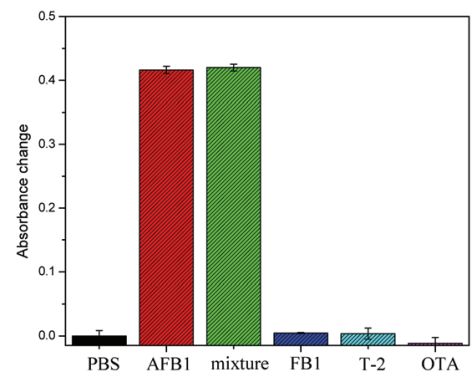

Fig. 9 Specificity of the developed assay towards AFB1 (1 ng mL $\left.\mathrm{m}^{-1}\right), \mathrm{FB} 1$ (20 ng mL $\left.\mathrm{mL}^{-1}\right), \mathrm{T}-2$ toxin $\left(20 \mathrm{ng} \mathrm{mL}^{-1}\right)$, OTA $\left(20 \mathrm{ng} \mathrm{mL}^{-1}\right)$ and their mixture with AFB1 $(n=3)$.

without and with gold enlargement. After gold staining, the signal response increased distinctly. Take $1 \mathrm{ng} \mathrm{mL}^{-1}$ AFB1 for example, the absorbance change is about 3.5 times of that without gold staining, which indicates that the sensitivity was indeed improved after gold enhancement. Moreover, the method is relatively fast, with a total assay time of $\sim 1 \mathrm{~h}$.

Further, the specificity of the developed immunoassay was evaluated using common mycotoxins as competitors, including FB1, T-2 toxin, OTA, and their mixture with AFB1. As shown in Fig. 9, a significant change in absorbance was only observed in the presence of AFB1, which demonstrated that the assay has a good selectivity toward AFB1 detection.

The feasibility of applying the established immunoassay for complex matrices was evaluated by the analysis of spiked maize samples. The results are summarized in Table 1. Satisfactory recoveries were obtained in the range of $90.7 \%$ to $115.1 \%$ with acceptable relative standard deviation (RSD). This analysis demonstrated that the established method could be used for quantitative monitoring of AFB1 in real samples.

The proposed method is simple, specific and highly sensitive. Compared with highly sensitive DNA amplification such as hybridization chain reaction $(\mathrm{HCR})^{40}$ and loop mediated isothermal amplification (LAMP),$^{41}$ the design and operation of the gold staining amplification method are much easier. Furthermore, the established method does not require expensive or challenging equipments. This strategy is very attractive for mycotoxin determination because (1) the preparation and the bio-functionalization of the nanoparticles is simple and generally applicable; (2) taking advantages of the amplification effect of catalytic deposition of gold together with the extremely high molar extinction coefficient of enlarged AuNPs, the gold enlargement-based colorimetric approach shows high sensitivity; (3) the use of magnetic

Table 1 Analysis of spiked maize samples $(n=3)$

\begin{tabular}{lccc}
\hline $\begin{array}{l}\text { In sample } \\
\left(\mu \mathrm{kg}^{-1}\right)\end{array}$ & $\begin{array}{l}\text { After dilution } \\
\left.(\mathrm{ng} \mathrm{L})^{-1}\right)\end{array}$ & $\begin{array}{l}\text { Detected value } \\
\left(\mathrm{ng} \mathrm{L}^{-1}\right)\end{array}$ & Recovery (\%) \\
\hline 4 & 50 & 51.5 & $103.0 \pm 8.2$ \\
8 & 100 & 115.1 & $115.1 \pm 13.2$ \\
20 & 250 & 255.6 & $102.2 \pm 6.9$ \\
40 & 500 & 453.3 & $90.7 \pm 2.1$
\end{tabular}


particles as solid carrier reduces the incubation time, and facilitates the rapid separation of immune complexes.

\section{Conclusions}

We have shown here the controlled growth of AuNPs using $\mathrm{CTAB}$ as stabilizing surfactant, $\mathrm{AA}$ as reducing agent, and $\mathrm{Na}_{2} \mathrm{~S}_{2} \mathrm{O}_{3}$ as stop reagent. Surface plasmon resonance signature of the enlarged AuNPs and the kinetics of the gold enlargement were monitored by UV-vis spectroscopy and RLS technique. The absorbance as well as RLS intensity of the enlarged AuNPs is highly dependent on the initial concentration of gold seeds. The controlled homogenous gold enlargement was then utilized for signal amplification in competitive immunoassay for the first time. High sensitivity and satisfactory recoveries in spiked maize samples were achieved for the detection of AFB1. The total assay is simple, specific, sensitive, and could be easily transferred to the detection of other toxins and organic pollutants.

\section{Acknowledgements}

The financial support of the China Scholarship Council is gratefully acknowledged.

\section{Notes and references}

1 E. C. Dreaden, A. M. Alkilany, X. Huang, C. J. Murphy and M. A. El-Sayed, Chem. Soc. Rev., 2012, 41, 2740-2779.

2 K. Saha, S. S. Agasti, C. Kim, X. Li and V. M. Rotello, Chem. Rev., 2012, 112, 2739-2779.

3 H. Jans and Q. Huo, Chem. Soc. Rev., 2012, 41, 2849-2866.

4 K. E. Sapsford, W. R. Algar, L. Berti, K. B. Gemmill, B. J. Casey, E. Oh, M. H. Stewart and I. L. Medintz, Chem. Rev., 2013, 113, 1904-2074.

5 L. Dykman and N. Khlebtsov, Chem. Soc. Rev., 2012, 41, 2256-2282.

6 J. Sun, Y. Xianyu and X. Jiang, Chem. Soc. Rev., 2014, 43, 6239-6253.

7 D. Vilela, M. C. González and A. Escarpa, Anal. Chim. Acta, 2012, 751, 24-43.

8 Y. Song, W. Wei and X. Qu, Adv. Mater., 2011, 23, 4215-4236.

9 X. Cao, Y. Ye and S. Liu, Anal. Biochem., 2011, 417, 1-16.

10 W. Lai, D. Tang, J. Zhuang, G. Chen and H.-H. Yang, Anal. Chem., 2014, 86, 5061-5068.

11 D. Tang, B. Liu, R. Niessner, P. Li and D. Knopp, Anal. Chem., 2013, 85, 10589-10596.

12 W.-H. Zhou, C.-L. Zhu, C.-H. Lu, X. Guo, F. Chen, H.-H. Yang and X. Wang, Chem. Commun., 2009, 6845-6847.

13 A. Ambrosi, F. Airo and A. Merkoçi, Anal. Chem., 2009, 82, 1151-1156.

14 L. Zhan, W. B. Wu, X. X. Yang and C. Z. Huang, New J. Chem., 2014, 38, 2935-2940.
15 W. Li, J. Li, W. Qiang, J. Xu and D. Xu, Analyst, 2013, 138, 760-766.

16 X. Que, D. Tang, B. Xia, M. Lu and D. Tang, Anal. Chim. Acta, 2014, 830, 42-48.

17 W. Li, W. Qiang, J. Li, H. Li, Y. Dong, Y. Zhao and D. Xu, Biosens. Bioelectron., 2014, 51, 219-224.

18 R. Liu, X. Liu, Y. Tang, L. Wu, X. Hou and Y. Lv, Anal. Chem., 2011, 83, 2330-2336.

19 R. Liu, Y. Zhang, S. Zhang, W. Qiu and Y. Gao, Appl. Spectrosc. Rev., 2014, 49, 121-138.

20 Z. Ma and S. F. Sui, Angew. Chem., Int. Ed., 2002, 41, 21762179.

21 D. Kim, W. L. Daniel and C. A. Mirkin, Anal. Chem., 2009, 81, 9183-9187.

22 A. Fan, C. Lau and J. Lu, Analyst, 2009, 134, 497-503.

23 V. Pavlov, Y. Xiao, B. Shlyahovsky and I. Willner, J. Am. Chem. Soc., 2004, 126, 11768-11769.

24 A. Fan, S. Cai, Z. Cao, C. Lau and J. Lu, Analyst, 2010, 135, 1400-1405.

25 Z. Zhan, C. Cao and S. J. Sim, Biosens. Bioelectron., 2010, 26, 511-516.

26 C. Cao, X. Li, J. Lee and S. J. Sim, Biosens. Bioelectron., 2009, 24, 1292-1297.

27 P. Li, Q. Zhang, D. Zhang, D. Guan, X. Ding, X. Liu, S. Fang, X. Wang and W. Zhang, Aflatoxins: Detect., Meas. Control, 2011, 11, 183-208.

28 W. S. Khayoon, B. Saad, C. B. Yan, N. H. Hashim, A. S. M. Ali, M. I. Salleh and B. Salleh, Food Chem., 2010, 118, 882-886.

29 A. Bacaloni, C. Cavaliere, F. Cucci, P. Foglia, R. Samperi and A. Laganà, J. Chromatogr. A, 2008, 1179, 182-189.

30 B. B. Dzantiev, N. A. Byzova, A. E. Urusov and A. V. Zherdev, TrAC, Trends Anal. Chem., 2014, 55, 81-93.

31 D. Tang, Y. Lin, Q. Zhou, Y. Lin, P. Li, R. Niessner and D. Knopp, Anal. Chem., 2014, 86, 11451-11458.

32 S. Oswald, X. Karsunke, R. Dietrich, E. Märtlbauer, R. Niessner and D. Knopp, Anal. Bioanal. Chem., 2013, 405, 6405-6415.

33 C. Cervino, E. Weber, D. Knopp and R. Niessner, J. Immunol. Methods, 2008, 329, 184-193.

34 X. Wang, R. Niessner and D. Knopp, Sensors, 2014, 14, 21535-21548.

35 Z. Khan, T. Singh, J. I. Hussain and A. A. Hashmi, Colloids Surf., B, 2013, 104, 11-17.

36 S. A. AL-Thabaiti, J. I. Hussain, A. A. Hashmi and Z. Khan, Can. Chem. Trans., 2013, 1, 238-252.

37 W. B. Wu, L. Zhan, J. Wang and C. Z. Huang, Anal. Methods, 2014, 6, 3779-3783.

38 D. Li, Y. Ying, J. Wu, R. Niessner and D. Knopp, Microchim. Acta, 2013, 180, 711-717.

39 D. Tang, Z. Zhong, R. Niessner and D. Knopp, Analyst, 2009, 134, 1554-1560.

40 B. Zhang, B. Liu, D. Tang, R. Niessner, G. Chen and D. Knopp, Anal. Chem., 2012, 84, 5392-5399.

41 M. Dou, D. C. Dominguez, X. Li, J. Sanchez and G. Scott, Anal. Chem., 2014, 86, 7978-7986. 